\title{
Molecular diagnostics of intestinal parasites in returning travellers
}

\author{
R. J. ten Hove - M. van Esbroeck • T. Vervoort • \\ J. van den Ende $\cdot$ L. van Lieshout $\cdot$ J. J. Verweij
}

Received: 25 November 2008 / Accepted: 31 March 2009/Published online: 5 May 2009

(C) The Author(s) 2009. This article is published with open access at Springerlink.com

\begin{abstract}
A new diagnostic strategy was assessed for the routine diagnosis of intestinal parasites in returning travellers and immigrants. Over a period of 13 months, unpreserved stool samples, patient characteristics and clinical data were collected from those attending a travel clinic. Stool samples were analysed on a daily basis by microscopic examination and antigen detection (i.e. care as usual), and compared with a weekly performed multiplex real-time polymerase chain reaction (PCR) analysis on Entamoeba histolytica, Giardia lamblia, Cryptosporidium and Strongyloides stercoralis. Microscopy and antigen assays of 2,591 stool samples showed E. histolytica, G. lamblia, Cryptosporidium and $S$. stercoralis in $0.3,4.7,0.5$ and $0.1 \%$ of the cases, respectively. These detection rates were increased using real-time PCR to $0.5,6.0,1.3$ and $0.8 \%$, respectively. The prevalence of ten additional pathogenic parasite species identified with microscopy was, at most, $0.5 \%$. A pre-selective decision tree based on travel history or gastro-intestinal complaints could not be made. With increased detection rates at a lower workload and the potential to extend with additional parasite targets combined with fully automated DNA isolation, molecular high-throughput screening could eventually replace microscopy to a large extent.
\end{abstract}

R. J. ten Hove $\cdot$ L. van Lieshout $\cdot$ J. J. Verweij $(\bowtie)$ Department of Parasitology, Leiden University Medical Centre, P.O. Box 9600, 2300 RC Leiden, The Netherlands e-mail: j.j.verweij@lumc.nl

M. van Esbroeck · T. Vervoort · J. van den Ende Department of Parasitology,

Prince Leopold Institute of Tropical Medicine, Nationalestraat 155 ,

2000 Antwerp, Belgium

\section{Introduction}

Over the past decennia, outbound tourism showed a worldwide increase and it is expected that this trend will continue in the future [1]. As a consequence, it is likely that a growing number of international travellers will consult a doctor after their return. Gastro-intestinal disorders are one of the main reasons for returning travellers to seek medical advice $[2,3]$. Moreover, several studies have shown that a large proportion of travellers and immigrants from tropical and subtropical countries harbour intestinal pathogens without clear gastro-intestinal problems [4-7]. Although this emphasises the need for a standard screening procedure for all travellers, the increasing numbers of samples will become a burden for routine diagnostic laboratories, especially during the peak periods of holidaying. Patients and diagnostic laboratories would, therefore, greatly benefit from the implementation of a sensitive high-throughput system for the screening of intestinal parasites.

Intestinal protozoan infections with Giardia lamblia and Cryptosporidium hominis/Cryptosporidium parvum are the main non-viral causes of diarrhoea in industrialised countries [8] and are even more frequently seen as the cause of gastro-intestinal complaints in returning travellers $[2,9,10]$. Although quite rare, the early diagnosis of Entamoeba histolytica is of vital importance because of the potential invasive character of this protozoan parasite. Intestinal helminth infections in travellers usually do not cause severe clinical complications. One important exception is Strongyloides stercoralis. Unlike other helminth infections, S. stercoralis is capable of maturing to the infective filariform stage in the intestinal lumen, causing auto-infection through larval penetration of the intestinal mucosa or the perianal skin. Even after decades, these chronic infections can develop into life-threatening hyper 
infections in immune depressed patients, especially those receiving immunosuppressive therapy with corticosteroids [11]. Laboratory diagnosis of schistosomiasis, which is frequently diagnosed in travellers, mainly depends on serology rather than on the microscopic detection of ova in stool and urine in this particular population [12].

Traditionally, the laboratory diagnosis of intestinal protozoan and helminth infections relies on the detection of trophozoites, cysts, eggs and larvae by microscopic stool examination. Although microscopy is considered to be the gold standard, it is labour-intensive and its diagnostic performance critically depends on well-trained microscopists. To improve sensitivity, multiple specimens and concentration procedures, as well as a variety of staining methods, are needed to achieve ample sensitivity [13, 14]. Enzyme immunoassays [15, 16] and direct fluorescentantibody assays [17] have been accepted as cost-effective alternative diagnostic methods for the detection of $G$. lamblia and Cryptosporidium in stools. The specific detection of E. histolytica cannot be achieved using microscopy alone, as cysts and (small) trophozoites of $E$. histolytica and non-pathogenic E. dispar are morphologically indistinguishable. Therefore, additional methods such as antigen detection or polymerase chain reaction (PCR) have to be employed [7]. The laboratory diagnosis of $S$. stercoralis is known to be problematic: the sensitivity and specificity of immunodiagnostic assays can vary considerably and the number of larvae in a stool sample can be very low. Multiple samples and concentration methods such as Baermann and copro-culture techniques are employed to increase the detection rates [18].

Although DNA-based methods for a variety of intestinal parasites have shown excellent sensitivity and specificity, until now, the introduction of these methods in daily laboratory practice has been limited. The introduction of real-time PCR combining several targets into one multiplex assay and the implementation of automated DNA-isolation methods offers the possibility of using DNA-based detection techniques in a high-throughput diagnostic approach. In a previous study, it was shown that in patients attending their general practitioner with gastro-intestinal problems, only two parasitic pathogens were found in such a population, i.e. G. lamblia and $C$. hominis/C. parvum [19]. The sensitivity of the multiplex real-time PCR proved to be much higher as compared to microscopy in detecting these two parasitic infections. In returning travellers, a larger variety of parasitic infections can be expected. Presently, one of the constraints of multiplex real-time PCR is the restriction in the number of parasitic targets that can be detected simultaneously. Therefore, a careful assessment is needed for the choice of parasitic targets when molecular diagnostic techniques are implemented.
In this prospective study, the performance of real-time PCR for the detection of E. histolytica, G. lamblia, $C$. hominis/C. parvum and S. stercoralis DNA in faeces was compared with current diagnostic tools, which consist of microscopy and antigen detection in stool samples from patients attending a travel clinic. Patient characteristics and clinical data were recorded to define a practical diagnostic strategy for the implementation of molecular methods in the routine laboratory diagnosis of intestinal parasitic infections in returning travellers and immigrants.

\section{Materials and methods}

Sample collection

Stool specimens were collected between April 2005 and May 2006 from outpatients attending the travel clinic of the Institute of Tropical Medicine (ITM) in Antwerp, Belgium. Age, gender, place of birth, travel history and gastrointestinal complaints were actively recorded by the attending physician and included in the database. Patients who were referred to the clinic specifically for HIV/AIDSrelated issues were not included in this study. A successive sample from the same patient analysed within 30 days of the preceding sample was excluded from the study. Subsequently, each sample was considered to have been obtained from a new patient. The protocol was approved by the ethical committee of the ITM. Patients gave informed consent and the data were rendered anonymous according to the Belgian legislation.

Stool specimens were collected according to the routine procedure of the ITM: patients were asked to fill an empty tube with stool, preferably at the clinic, or otherwise to send it to the diagnostic laboratory of the ITM by regular mail. Aliquots of the samples were stored in the refrigerator until they were sent to the Leiden University Medical Centre (LUMC) for real-time PCR analysis on a weekly transport by regular mail. Molecular diagnostics were performed immediately after the arrival of the samples and the results were generated blinded to the result of the conventional stool examination at the ITM.

\section{Microscopy and copro-antigen detection}

Microscopic examination for the presence of ova and cysts was performed daily according to the standard routine procedures at the diagnostic laboratory of the ITM by the examination of unstained and iodine-stained direct smears with saline and unstained and iodine-stained wet mounts after formalin-ether concentration [20]. Additionally, carbol-fuchsine staining was performed on formalin-ether concentrates for the detection of coccidian parasites [21]. 
Both pathogenic and non-pathogenic parasites were recorded.

Copro-antigen enzyme-linked immunosorbent assays (ELISAs) for the detection of Giardia, Cryptosporidium and E. histolytica/E. dispar (ProSpecT, Remel, Lenexa, Kansas, USA) were performed on all specimens. The $E$. histolytica-specific copro-antigen tests (TechLab, Blacksburg, VA, USA) was performed if microscopic examination revealed E. histolytica/E. dispar cysts and/or if the $E$. histolytica/E. dispar antigen ELISA showed a positive result. In the diagnostic procedure at the ITM before the start of this study, E. histolytica/E. dispar-positive samples were routinely tested with the E. histolytica/E. dispar (HD) PCR [22]. As in this study, E. histolytica PCR would be performed as part of the E. histolytica/G. lamblia/Cryptosporidium (HGC) PCR on all samples; HD PCR was performed only on the microscopy or antigen E. histolytical E. dispar-positive samples retrospectively.

In clinically suspected strongyloidiasis cases, Baermann's method was requested to detect $S$. stercoralis larvae $[23,24]$. The detection of Enterobius vermicularis eggs was performed on demand using the scotch-tape method [25].

\section{DNA isolation}

For DNA isolation, $200 \mu \mathrm{l}$ of faeces suspension (approximately $0.5 \mathrm{~g} / \mathrm{ml}$ PBS containing $2 \%$ polyvinylpolypyrrolidone [PVPP; Sigma]) was heated for $10 \mathrm{~min}$ at $100^{\circ} \mathrm{C}$. After sodium-dodecyl-sulphate-proteinase $\mathrm{K}$ treatment (overnight at $55^{\circ} \mathrm{C}$ ), DNA was isolated with QIAamp Tissue Kit spin columns (QIAgen, Hilden, Germany) [26]. In each sample, $10^{3}$ plaque-forming units $(\mathrm{PFU}) / \mathrm{ml}$ phocin herpes virus 1 (PhHV-1) was added within the isolation lysis buffer, to serve as an internal control for the isolation, amplification and detection of the multiplex real-time PCR assays [27].

\section{PCR amplification and detection}

E. histolytica, G. lamblia and C. hominis/C. parvum multiplex real-time PCR including PhHV-1 as an internal control (HGC PCR) was performed as described previously with some modifications [28]. S. stercoralis DNA amplification was performed in a separate assay, also including PhHV-1 as an internal control [29].

Amplification and detection reactions for HGC PCR were performed in a volume of $25 \mu$ containing PCR buffer (Hotstar master mix, QIAgen, Venlo, The Netherlands), $5 \mathrm{mM} \mathrm{MgCl}_{2}, 2.5 \mu \mathrm{g}$ bovine serum albumin (BSA) (Roche Diagnostics, Almere, The Netherlands), 3.13 pmol of each E. histolytica-specific primer, 1.25 pmol VIC-labelled MGB probe for E. histolytica (Applied Biosystems, Warrington, UK), $3.13 \mathrm{pmol}$ of each $G$. lamblia primer, $2.5 \mathrm{pmol} G$. lamblia-specific FAM-double-labelled probe (Biolegio, Mal- den, The Netherlands), $12.5 \mathrm{pmol}$ of each $C$. hominis/C. parvum-specific primer, $2.5 \mathrm{pmol}$ of Texas Red doublelabelled probe for $C$. hominis/C. parvum, $3.75 \mathrm{pmol}$ of each PhHV-1-specific primer, 2.5 pmol of PhHV-1-specific Cy5double-labelled probe [27] and $5 \mu 1$ of the DNA sample. Amplification consisted of $15 \mathrm{~min}$ at $95^{\circ} \mathrm{C}$ followed by 50 cycles of $15 \mathrm{~s}$ at $95^{\circ} \mathrm{C}, 30 \mathrm{~s}$ at $60^{\circ} \mathrm{C}$ and $30 \mathrm{~s}$ at $72^{\circ} \mathrm{C}$. Amplification, detection and data analysis for $\mathrm{HGC}$ real-time PCR was performed with the i-Cycler real-time detection system (BioRad, Veenendaal, The Netherlands).

The amplification and detection of $S$. stercoralis-specific DNA was performed with 5 pmol of each S. stercoralisspecific primers, $2.5 \mathrm{pmol}$ of $S$. stercoralis-specific FAMdouble-labelled probe (Biolegio), 3.75 pmol of each PhHV-1-specific primer, 2.5 pmol of PhHV-1-specific Cy5double-labelled probe [27], PCR buffer (Hotstar master mix, QIAgen), $5 \mathrm{mM} \mathrm{MgCl} 2,2.5 \mu \mathrm{g}$ BSA and $5 \mu \mathrm{l}$ of the DNA sample in a final volume of $25 \mu 1$ [29]. Amplification consisted of $15 \mathrm{~min}$ at $95^{\circ} \mathrm{C}$ followed by 50 cycles of $15 \mathrm{~s}$ at $95^{\circ} \mathrm{C}$ and $60 \mathrm{~s}$ at $60^{\circ} \mathrm{C}$. Amplification, detection and data analysis for S. stercoralis real-time PCR was performed with the Applied Biosystems 7500 Real-Time PCR System and Sequence Detection Software version 1.2.2.

At the end of the study period, E. histolytica/E. disparpositive samples with microscopy- and/or copro-antigen detection HD PCR [22] was performed, retrospectively. Amplification and detection was performed with 3.125 pmol of each E. histolytica/E. dispar-specific primers, $2.5 \mathrm{pmol}$ of $E$. dispar-specific FAM-labelled MGB probe (Applied Biosystems) [22], 3.75 pmol of each PhHV-1-specific primer, 2.5 pmol of PhHV-1-specific Cy5-double-labelled probe [27], PCR buffer (HotStar master mix, QIAgen), $5 \mathrm{mM} \mathrm{MgCl} 2,2.5 \mu \mathrm{g}$ BSA and $5 \mu \mathrm{l}$ of the DNA sample in a final volume of $25 \mu \mathrm{l}$. Amplification consisted of $15 \mathrm{~min}$ at $95^{\circ} \mathrm{C}, 30 \mathrm{~s}$ at $60^{\circ} \mathrm{C}$ and $30 \mathrm{~s}$ at $72^{\circ} \mathrm{C}$. Amplification, detection and data analysis for HD real-time PCR was performed with the i-Cycler real-time detection system.

High cycle threshold values (Ct-values) obtained by realtime PCR are considered to be less reproducible due to very low copy numbers of the specific target. Therefore, the PCR of samples revealing Ct-values above 36 were repeated. Also, the amplification was considered to be hampered by faecal inhibitory factors if the expected Ctvalue of 33 in the PhHV-1-specific PCR was increased with more than 3.3 cycles. Samples were recorded negative for the specific target if the positive (high) Ct-value could not be reproduced or were excluded if the amplification remained inhibited.

\section{Data analysis}

The results of the microscopic examinations, copro-antigen tests and real-time PCR analysis were stored and grouped in 
an Access database (Microsoft, Redmond, WA, USA). Data included patient's demographic information, travel history and clinical presentation, the laboratory results of the travel clinic in Antwerpen and the real-time PCR results of the laboratory for parasitology in Leiden. Travel destinations were classified according to geographical regions: Central America, South America, Asia, Northern Africa and subSaharan Africa were considered as high-risk areas and others (including Europe, United States of America and Australia) as being at a low risk of contracting intestinal infections. World travellers or sailors were also considered to have a high risk of contracting intestinal infections.

Analysis was done with SPSS 14.0 (SPSS Inc., Chicago, IL, USA). Continuous variables were described by the range and median of all positive cases and were compared between groups by the Mann-Whitney rank-sum test. For this purpose, zero values in Ct-values were redefined as 50 . Statistical significance was considered at $P<0.05$. The Chisquare distribution for the risk of acquiring pathogenic intestinal parasites, recorded as high- or low-risk travel destination, or for the presence of gastro-intestinal complaints, was calculated as a proportion of parasite infections detected by any of the used diagnostic techniques.

\section{Results}

\section{Study group}

Over a period of 13 months, 2,709 samples were collected and analysed (Fig. 1). One hundred and eighteen samples were excluded from the study population as the time interval after the preceding sample of the same patient was less than 30 days. The final number of participants included in this study was 2,591 . PhHV internal control was amplified within the correct $\mathrm{Ct}$ range in all samples; therefore, no samples were excluded due to inhibition. The travellers' ages varied from newborn to 85 years (median 36) and $55.5 \%$ of the patient cohort was of the male gender. Travel to 142 different countries or areas was mentioned, most frequently to sub-Saharan Africa (50.9\%). The majority of travellers were born in Europe (73.7\%) or on the African continent (19.0\%). Gastro-intestinal complaints were mentioned by $897(34.6 \%)$ patients and are listed in more detail in Table 1, together with the other patient's characteristics.

\section{Diagnosis of Entamoeba histolytica/Entamoeba dispar, Giardia lamblia and Cryptosporidium spp.}

The results of microscopy, copro-antigen and real-time PCR for all faecal parasites are summarised in Table 2. E. histolytica/E. dispar cysts and trophozoites were detected by microscopy in 99 cases. Fifteen additional cases were detected with $E$ histolytica/E. dispar copro-antigen ELISA. The presence of E. histolytica was confirmed with the species-specific copro-antigen ELISA in seven cases. In one of these seven samples, trophozoites with ingested red blood cells were seen in the direct smear, representing the only distinctive morphologic features of E. histolytica. E. histolytica-specific amplification was detected using the HGC PCR in 13 samples, with Ct-values between 20.7 and 38.7 (median 31.3). Retrospective analysis with HD PCR of 114 E. histolyticalE. dispar microscopy and/or coproantigen-positive samples confirmed 88 samples as $E$. dispar and 14 samples as E. histolytica, whereas HD PCR remained negative for both targets in 12 samples.

G. lamblia cysts and/or trophozoites were detected with microscopy in 95 cases and 121 cases revealed a positive result in the Giardia antigen ELISA. The Giardia antigen
Fig. 1 Number of all collected stool samples $(n=2,709)$ on a weekly basis between April 2005 and May 2006. Sharp increases of sample collections due to holidays are observed in August 2005 and at beginning of January 2006

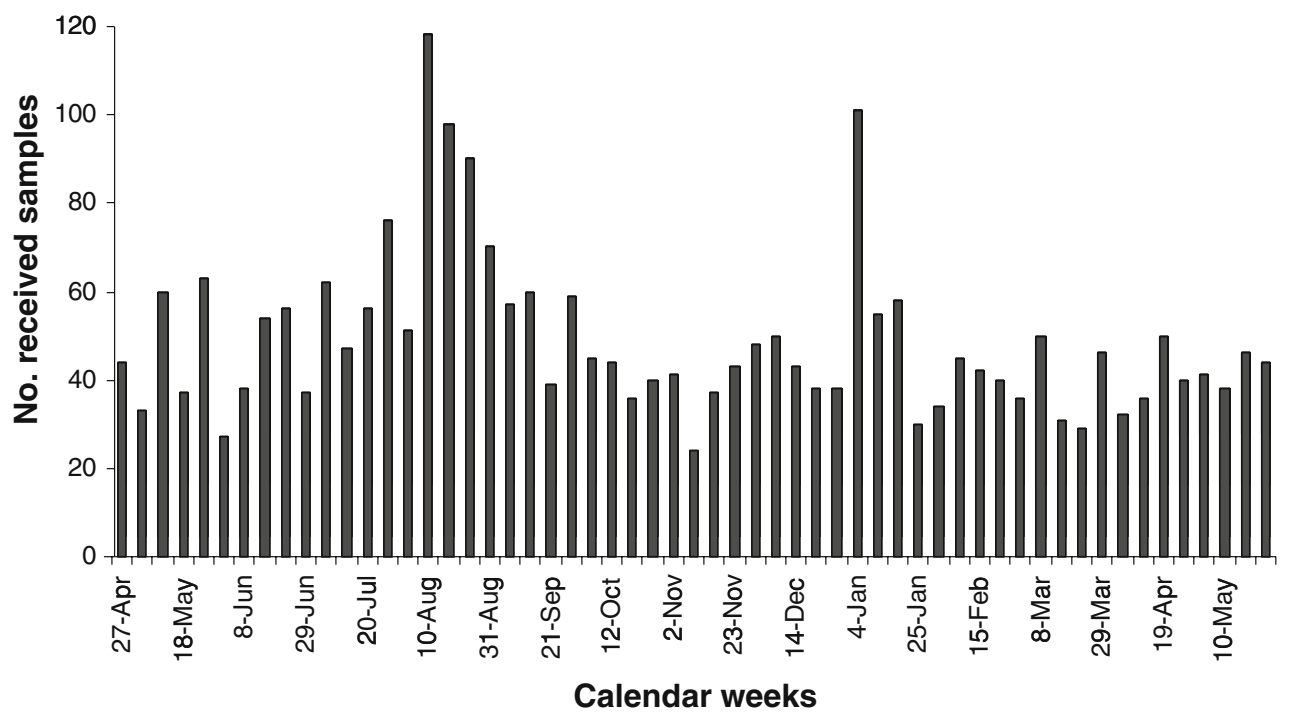


Table 1 Epidemiological and clinical characteristics of the study population $(n=2,591)$

\begin{tabular}{|c|c|}
\hline Characteristics & $n(\%)$ \\
\hline \multicolumn{2}{|l|}{ Patient characteristics } \\
\hline Male & $1,437(55.5)$ \\
\hline Age range, median, years & $0-85(36.0)$ \\
\hline Children, age $<15$ years & $161(6.2)$ \\
\hline Elderly, age $>59$ years & $259(10.0)$ \\
\hline Born in Europe & $1,910(73.7)$ \\
\hline Born in Africa & $491(19.0)$ \\
\hline Other/unknown country of birth & $190(7.4)$ \\
\hline \multicolumn{2}{|l|}{ Last visited region } \\
\hline Low-risk areas & $98(3.8)$ \\
\hline Central America & $99(3.8)$ \\
\hline South America & $130(5.0)$ \\
\hline Asia & $458(17.7)$ \\
\hline North Africa & $102(3.9)$ \\
\hline Sub-Saharan Africa & $1,318(50.9)$ \\
\hline World travellers & $17(0.7)$ \\
\hline Unknown & $369(14.2)$ \\
\hline \multicolumn{2}{|l|}{ In Belgium since } \\
\hline$<1$ month & $1,143(44.1)$ \\
\hline $1-6$ months & $579(22.3)$ \\
\hline 6-12 months & $170(6.6)$ \\
\hline$>12$ months & $476(18.4)$ \\
\hline Unknown & $223(8.6)$ \\
\hline \multicolumn{2}{|l|}{ Gastro-intestinal complaints } \\
\hline No gastro-intestinal complaints & $1,692(65.3)$ \\
\hline Diarrhoea, flatulence, pain and/or nausea & $897(34.6)$ \\
\hline Unknown & $2(0.1)$ \\
\hline \multicolumn{2}{|l|}{ Gastro-intestinal complaints specified } \\
\hline Diarrhoea & $635(24.5)$ \\
\hline Flatulence & $380(14.7)$ \\
\hline Cramps/pain & $608(23,5)$ \\
\hline Nausea & $280(10,8)$ \\
\hline \multicolumn{2}{|l|}{ Duration of complaints } \\
\hline$<2$ weeks & $334(12.9)$ \\
\hline$>2$ weeks & $563(21.7)$ \\
\hline \multicolumn{2}{|l|}{ Stool consistency } \\
\hline Formed & $1,765(68.1)$ \\
\hline Unformed & $765(29.5)$ \\
\hline Watery & $49(1.9)$ \\
\hline Unknown & $12(0.5)$ \\
\hline Bloody & $37(1.4)$ \\
\hline Mucous & $1(<0.1)$ \\
\hline
\end{tabular}

ELISA was positive in 28 microscopy-negative cases and did not confirm two microscopy-positive samples. $G$. lamblia-specific amplification was detected in 149 samples with Ct-values between 17.0 and 44.7 (median 29.8).
Cryptosporidium oocysts were detected in 12 carbolfuchsine-stained samples. The copro-antigen tests confirmed all Cryptosporidium microscopy-positive samples and two additional samples were found positive in the Cryptosporidium antigen ELISA. C. hominis/C. parvumspecific amplification was detected in 31 samples with Ctvalues between 24.4 and 39.5 (median 35.6).

Ct-values of samples positive with microscopy and/or copro-antigen tests were significantly lower as compared to samples with a negative result in the conventional methods $(P<0.001)$ for both $G$. lamblia and C. hominis/C. parvum (data not shown).

Discrepancies between real-time HGC PCR and conventional methods were observed in the following cases. $E$. histolytica real-time PCR was negative in one sample in which $E$. histolytica/E. dispar cysts were observed with microscopy and both copro-antigen ELISAs tested positive. Giardia real-time PCR remained negative in two cases in which cysts were seen in microscopy and in five cases in which only the copro-antigen assay tested positive. Cryptosporidium real-time PCR remained negative in two samples in which only the Cryptosporidium copro-antigen tested positive. The HGC multiplex real-time PCR detected a total of 49 additional cases which were not detected with microscopy and antigen tests.

\section{Strongyloides stercoralis}

Rhabditiform S. stercoralis larvae were detected with microscopy on concentrated smears in three samples. In one of these samples, the number of larvae was exceptionally high and the larvae were also detected in direct smear. Baermann's method was performed on stool samples from 121 clinically suspected cases and was only found to be positive in the same sample that was found to be positive in the direct smear. S. stercoralis-specific amplification was detected in all three microscopy-positive samples and in 18 additional samples with Ct-values ranging from 24.5 to 39.5 (median of 33.3).

Other parasitic infections

Microscopy revealed 55 additional pathogenic parasites that were not targeted with real-time PCR. Mixed infections with two pathogenic parasite species were observed in 18 patients (Table 3). Non-pathogenic parasites as seen in direct smears and wet mounts after concentration are also summarised in Table 2.

Travel history, symptoms and intestinal parasites

Detected parasitic infections in relation to travel destination and gastro-intestinal complaints are summarised in Table 4. 
Table 2 Intestinal parasites in the study population $(n=2,591)$ as detected with microscopy, copro-antigen test and real-time polymerase chain reaction (PCR)
${ }^{\mathrm{a}}$ Direct microscopy, after formalin-ether concentration, scotch tape test for $E$. vermicularis, Baermann test for S. stercoralis, and carbolfuchsine staining for Cryptosporidium spp.

${ }^{\mathrm{b}}$ Observed hematophagous trophozoites

${ }^{\mathrm{c}}$ E. histolytica / E. dispar coproantigen test (ProSpect, Remel, Lenexa, Kansas, USA)

${ }^{\mathrm{d}}$ E. histolytica specific coproantigen test (TechLab, Blackburg, Virginia, USA)

\begin{tabular}{|c|c|c|c|c|}
\hline Pathogens & Total detected & Microscopy $^{\mathrm{a}}$ & Antigen & Real-time PCR \\
\hline E. histolytica/E. dispar & 114 & 99 & $90^{\mathrm{c}}$ & - \\
\hline E. histolytica & 14 & $1^{\mathrm{b}}$ & $7^{\mathrm{d}}$ & 13 \\
\hline G. lamblia & 156 & 95 & 121 & 149 \\
\hline Cryptosporidium spp. & 33 & 12 & 14 & 31 \\
\hline S. stercoralis & 21 & 3 & - & 21 \\
\hline T. trichiura & 14 & 14 & - & - \\
\hline Hookworm & 10 & 10 & - & - \\
\hline A. lumbricoides & 8 & 8 & - & - \\
\hline Trichostrongylus spp. & 3 & 3 & - & - \\
\hline E. vermicularis & 1 & 1 & - & - \\
\hline S. mansoni & 11 & 11 & - & - \\
\hline S. haematobium & 1 & 1 & - & - \\
\hline Taenia spp. & 1 & 1 & - & - \\
\hline C. cayetanensis & 4 & 4 & - & - \\
\hline I. belli & 2 & 2 & - & - \\
\hline \multicolumn{5}{|l|}{ Non-pathogens } \\
\hline E. coli & 246 & 246 & - & - \\
\hline B. hominis & 220 & 220 & - & - \\
\hline E. nana & 139 & 139 & - & - \\
\hline E. hartmanii & 59 & 59 & - & - \\
\hline I. butschlii & 26 & 26 & - & - \\
\hline C. mesnilii & 23 & 23 & - & - \\
\hline S. hominis & 6 & 6 & - & - \\
\hline
\end{tabular}

The majority $(69.2 \%)$ of the study participants travelled to areas where the exposure risk to intestinal parasites is considered to be high. Among those with high-risk travel destinations, infection rates were not significantly higher for any of the pathogenic parasites compared with those

Table 3 Observed double infections of pathogenic parasites in stool samples $(n=2,591)$, detected by microscopy, copro-antigen detection and/or real-time PCR

\begin{tabular}{ll}
\hline Combination of species found & Number of samples \\
\hline E. histolytica and G. lamblia & 1 \\
E. histolytica and Cryptosporidium spp. & 1 \\
E. histolytica and Hookworm & 1 \\
G. lamblia and Cryptosporidium spp. & 4 \\
G. lamblia and S. stercoralis & 2 \\
G. lamblia and T. trichiura & 2 \\
G. lamblia and Trichostrongylus spp. & 1 \\
Cryptosporidium spp. and T. trichiura & 1 \\
S. stercoralis and A. lumbricoides & 1 \\
A. lumbricoides and E. vermicularis & 1 \\
A. lumbricoides and hookworm & 1 \\
Hookworm and T. trichiura & 1 \\
Trichostrongylus spp. and S. mansoni & 1 \\
\hline
\end{tabular}

who travelled to low-risk areas. Gastro-intestinal complaints were correlated with $G$. lamblia- and $C$. hominis $/ C$. parvum detection $(P<0.001)$, but not with the detection of E. histolytica, S. stercoralis or other pathogenic parasitic infections.

\section{Discussion}

The diagnosis of intestinal parasitic infections in returned travellers traditionally relies on time-consuming analyses by experienced microscopists. Nowadays, the increasing number of travellers to a variety of exotic countries calls for new diagnostic approaches for the efficient processing of samples. In this study, multiplex real-time PCR was compared with the routine approach of microscopy and antigen-based methods, focussing on four target parasites, $E$. histolytica, G. lamblia, Cryptosporidium and S. stercoralis, respectively.

The results showed PCR to be more sensitive for the specific detection of E. histolytica, G. lamblia, C. hominis/ $C$. parvum and $S$. stercoralis. Only a few unexplained discrepancies between the different detection methods were seen. These were mostly cases in which only the antigen test was positive. In one sample, E. histolytica/E. dispar cysts were seen with microscopy and both antigen ELISAs 
Table 4 Detected intestinal parasites in the study population $(n=2,591)$ by real-time PCR, microscopy and/or antigen test listed in numbers and proportion of travel destination or the presence of gastro-intestinal (GI) complaints

\begin{tabular}{llllll}
\hline & \multicolumn{2}{l}{ PCR/microscopy/ag } & & Microscopy \\
\cline { 2 - 5 } & E. histolytica & G. lamblia & Cryptosporidium & S. stercoralis & additional pathogenic parasites \\
\hline High-risk travel pop. ${ }^{\mathrm{a}}, n=1,793(100 \%)$ & $10(0.6 \%)$ & $121(6.7 \%)$ & $26(1.5 \%)$ & $16(0.9 \%)$ & $37(2.1 \%)$ \\
Low-risk travel pop. ${ }^{\mathrm{b}}, n=429(100 \%)$ & $3(0.7 \%)$ & $19(4.4 \%)$ & $4(0.9 \%)$ & $1(0.2 \%)$ & $4(0.9 \%)$ \\
Destination unknown, $n=369(100 \%)$ & $1(0.3 \%)$ & $16(4.3 \%)$ & $3(0.8 \%)$ & $4(1.1 \%)$ & $14(3.8 \%)$ \\
With GI complaints, $n=897(100 \%)$ & $7(0.8 \%)$ & $76(8.5 \%)$ & $25(2.8 \%)$ & $4(0.4 \%)$ & $18(2.0 \%)$ \\
No complaints, $n=1,694(100 \%)^{\mathrm{c}}$ & $7(0.4 \%)$ & $80(4.7 \%)$ & $8(0.5 \%)$ & $17(1.0 \%)$ & $37(2.2 \%)^{\mathrm{d}}$ \\
Total, $n=2,591(100 \%)$ & $14(0.5 \%)$ & $156(6.0 \%)$ & $33(1.3 \%)$ & $21(0.8 \%)$ & $55(2.1 \%)$ \\
\hline
\end{tabular}

${ }^{a}$ Sub-Saharan Africa, Asia, world travellers

${ }^{\mathrm{b}}$ Europe, America, Australia, North Africa

${ }^{\mathrm{c}}$ Two cases with complaints not registered are added to the traveller group without gastro-intestinal complaints

${ }^{\mathrm{d}}$ Group contains four travellers with double infections

showed a positive result; however, E. histolytica-specific amplification was not successful in the initial HGC PCR. A positive result was obtained when the HGC real-time PCR was repeated, as well as in the HD real-time PCR. Internal and positive controls in the initial real-time PCR analysis were correct and the reason for the negative outcome of the first real-time PCR analysis remains unclear. This one case is discordant with the proven higher sensitivity of $E$. histolytica real-time PCR compared to stool antigen assays in a non-endemic setting [30, 31].

It is well known that the laboratory diagnosis of $S$. stercoralis requires multiple fresh stool samples using concentration techniques and/or copro-culture in order to improve detection rates [32]. In this study, the Baermann concentration technique was requested by the clinician in case of clinical suspicion, yet many Baermann tests were omitted because, for example, the sample was too old (had been sent by mail). Although the positive results of the $S$. stercoralis real-time PCR was confirmed by microscopy in three cases only, the other positive results were supported by serology $(n=7)$, eosinophilia $(n=5)$ and/or clinical presentation $(n=4)$ in the majority of cases.

In this study population, ten additional pathogenic parasite species detected by microscopy were missed with only four targeted parasite species in the real-time PCR. However, the prevalence of each detected species was only $0.5 \%$ at most and the clinical significance of the majority of these parasitic infections is limited. Most of these infections were helminths, of which the eggs could be easily found with microscopy at low magnification of an unstained wet mount of the concentrated sample. Schistosomiasis is probably the most relevant and also probably the most underestimated infection in this study, as the laboratory diagnosis of Schistosoma infections in travellers relies mainly on serology [33]. The implementation of a Schisto- soma real-time PCR [34] might be a worthwhile addition to a molecular diagnostic panel; however, its performance in a routine setting still needs further evaluation. Finally, two remaining protozoan infections, Isospora belli and Cyclospora cayetanensis, are important candidates as additional real-time PCR targets for patients returning from high-risk areas [35-37].

Designing an efficient diagnostic strategy requires a thorough exploration of possible predictors for parasite exposure in patient groups. A rationale for a specific diagnostic approach in a travel population is less evident compared with a patient group without extensive travel background [19]. In the travel population of this study, only a minority (34.6\%) mentioned gastro-intestinal complaints. Stool examination was also performed as part of a routine screening procedure. The presence of gastro-intestinal complaints was a significant predictor only for the presence of G. lamblia and C. hominis/C. parvum. In the cases without complaints, however, the prevalence of $G$. lamblia was still higher than any of the other pathogenic parasites in the total population. Furthermore, travel destinations were of little predictive value for the presence of any of the parasitic infections. In conclusion, travel destinations or gastro-intestinal complaints did not provide a diagnostic key towards specific pathogenic parasites in this study. As already seen in other studies, an exception can be made for the diagnosis of schistosomiasis, as cases are mainly found in travellers returning from Africa $[6,33]$.

The overall low prevalence of intestinal parasitic infections found in this study emphasises the need for a rapid, sensitive and simple screening assay for the most important parasitic infections in all returned travellers, disregarding their travel history and the presence of gastro-intestinal complaints, which agrees with recommendations made in other studies $[6,38,39]$. The low 
prevalence of additionally detected parasites raises doubts about the cost benefit of elaborate microscopic analyses of stool samples. An in-depth study on the cost per detected parasite or profit per diagnosis by different technical approaches will further elucidate the most beneficial strategy for a laboratory. For example, the staff utilisation for stool analysis with conventional techniques consists in this study of approximately one full-time equivalent (FTE) compared to 0.3 FTE for stool DNA isolation and real-time PCR analysis. Moreover, a fully automated DNA isolation process and extension of additional molecular targets on the already isolated DNA will have considerable impact on the cost-efficiency of the diagnostic procedures. Already, a growing number of routine diagnostic laboratories are implementing multiplex real-time PCR for the detection of intestinal microorganisms [19, 40, 41]. These standard PCR assays can be extended specifically for the travel population with one or more additional multiplex real-time PCR panels for an overlap of the most important intestinal parasitic infections.

Real-time PCR has proved to be a highly sensitive and specific technique for the detection of the majority of intestinal parasites found in returning travellers presenting at a travel clinic with and without gastro-intestinal complaints. The diagnostic approach for the detection of intestinal parasites in returning travellers in a routine diagnostic laboratory could be limited to real-time PCR for E. histolytica, G. lamblia, Cryptosporidium spp. and $S$. stercoralis. In addition, Schistosoma serology should be performed for travellers to Africa [6]. This approach could be complemented with additional multiplex PCRs and/or a quick microscopic examination for the presence of helminth eggs. Fully automated procedures and combination with additional targets might replace microscopy in the future to a large extent.

Acknowledgements We thank John Ackers for reviewing the manuscript.

Open Access This article is distributed under the terms of the Creative Commons Attribution Noncommercial License which permits any noncommercial use, distribution, and reproduction in any medium, provided the original author(s) and source are credited.

\section{References}

1. World Tourism Organization (2001) Global Forecast and Profiles of Market Segments. Tourism: 2020 Vision, Vol 7. World Tourism Organization, Madrid, Spain. ISBN 978-92-844-0466-7

2. Freedman DO, Weld LH, Kozarsky PE, Fisk T, Robins R, von Sonnenburg F, Keystone JS, Pandey P, Cetron MS; GeoSentinel Surveillance Network (2006) Spectrum of disease and relation to place of exposure among ill returned travelers. N Engl J Med 354:119-130. doi:10.1056/NEJMoa051331
3. Ansart S, Perez L, Vergely O, Danis M, Bricaire F, Caumes E (2005) Illnesses in travelers returning from the tropics: a prospective study of 622 patients. J Travel Med 12:312-318

4. Caruana SR, Kelly HA, Ngeow JY, Ryan NJ, Bennett CM, Chea L, Nuon S, Bak N, Skull SA, Biggs BA (2006) Undiagnosed and potentially lethal parasite infections among immigrants and refugees in Australia. J Travel Med 13:233-239. doi:10.1111/ j.1708-8305.2006.00045.x

5. Saiman L, Aronson J, Zhou J, Gomez-Duarte C, Gabriel PS, Alonso M, Maloney S, Schulte J (2001) Prevalence of infectious diseases among internationally adopted children. Pediatrics 108:608-612. doi:10.1542/peds.108.3.608

6. Whitty CJ, Carroll B, Armstrong M, Dow C, Snashall D, Marshall T, Chiodini PL (2000) Utility of history, examination and laboratory tests in screening those returning to Europe from the tropics for parasitic infection. Trop Med Int Health 5:818-823. doi:10.1046/j.1365-3156.2000.00642.x

7. Fotedar R, Stark D, Beebe N, Marriott D, Ellis J, Harkness J (2007) Laboratory diagnostic techniques for Entamoeba species. Clin Microbiol Rev 20:511-532. doi:10.1128/CMR.00004-07

8. de Wit MAS, Koopmans MPG, Kortbeek LM, Wannet WJB, Vinjé J, van Leusden F, Bartelds AIM, van Duynhoven YTHP (2001) Sensor, a population-based cohort study on gastroenteritis in the Netherlands: incidence and etiology. Am J Epidemiol 154:666-674. doi:10.1093/aje/154.7.666

9. Thielman NM, Guerrant RL (1998) Persistent diarrhea in the returned traveler. Infect Dis Clin North Am 12:489-501. doi:10.1016/S0891-5520(05)70015-5

10. Okhuysen PC (2001) Traveler's diarrhea due to intestinal protozoa. Clin Infect Dis 33:110-114. doi:10.1086/320894

11. Concha R, Harrington W Jr, Rogers AI (2005) Intestinal strongyloidiasis: recognition, management, and determinants of outcome. J Clin Gastroenterol 39:203-211. doi:10.1097/01. mcg.0000152779.68900.33

12. Bottieau E, Clerinx J, Van den Enden E, Van Esbroeck M, Colebunders R, Van Gompel A, Van den Ende J (2007) Fever after a stay in the tropics: diagnostic predictors of the leading tropical conditions. Medicine 86:18-25. doi:10.1097/MD.0b013e $3180305 \mathrm{c} 48$

13. Branda JA, Lin TY, Rosenberg ES, Halpern EF, Ferraro MJ (2006) A rational approach to the stool ova and parasite examination. Clin Infect Dis 42:972-978. doi:10.1086/500937

14. Morgan UM, Thompson RC (1998) Molecular detection of parasitic protozoa. Parasitology 117(Suppl):S73-S85

15. Weitzel T, Dittrich S, Möhl I, Adusu E, Jelinek T (2006) Evaluation of seven commercial antigen detection tests for Giardia and Cryptosporidium in stool samples. Clin Microbiol Infect 12:656-659. doi:10.1111/j.1469-0691.2006.01457.x

16. Katanik MT, Schneider SK, Rosenblatt JE, Hall GS, Procop GW (2001) Evaluation of ColorPAC Giardia/Cryptosporidium rapid assay and ProSpecT Giardia/Cryptosporidium microplate assay for detection of Giardia and Cryptosporidium in fecal specimens. J Clin Microbiol 39:4523-4525. doi:10.1128/JCM.39.12.45234525.2001

17. Garcia LS, Shimizu RY (1997) Evaluation of nine immunoassay kits (enzyme immunoassay and direct fluorescence) for detection of Giardia lamblia and Cryptosporidium parvum in human fecal specimens. J Clin Microbiol 35:1526-1529

18. Steinmann P, Zhou XN, Du ZW, Jiang JY, Wang LB, Wang XZ, Li LH, Marti H, Utzinger J (2007) Occurrence of Strongyloides stercoralis in Yunnan Province, China, and comparison of diagnostic methods. PLoS Negl Trop Dis 1:e75. doi:10.1371/ journal.pntd.0000075

19. ten Hove R, Schuurman T, Kooistra M, Möller L, van Lieshout L, Verweij JJ (2007) Detection of diarrhoea-causing protozoa in general practice patients in The Netherlands by multiplex real- 
time PCR. Clin Microbiol Infect 13:1001-1007. doi:10.1111/ j.1469-0691.2007.01788.x

20. Loughlin EH, Spitz SH (1949) Diagnosis of helminthiasis. JAMA 139:997-1000

21. Heine J (1982) A simple technic for the demonstration of cryptosporidia in feces. Zentralbl Veterinarmed B 29:324-327

22. Verweij JJ, Oostvogel F, Brienen EA, Nang-Beifubah A, Ziem J, Polderman AM (2003) Short communication: Prevalence of Entamoeba histolytica and Entamoeba dispar in northern Ghana. Trop Med Int Health 8:1153-1156. doi:10.1046/j.1360-2276.2003.01145.x

23. Jones CA, Abadie SH (1954) Studies in human strongyloidiasis. II. A comparison of the efficiency of diagnosis by examination of feces and duodenal fluid. Am J Clin Pathol 24:1154-1158

24. Pereira Lima J, Delgado PG (1961) Diagnosis of strongyloidiasis: importance of Baermann's method. Am J Dig Dis 6:899-904. doi:10.1007/BF02231086

25. Graham CF (1941) A device for the diagnosis of Enterobius infection. Am J Trop Med Hyg 21:159-161

26. Verweij JJ, Blotkamp J, Brienen EAT, Aguirre A, Polderman AM (2000) Differentiation of Entamoeba histolytica and Entamoeba dispar cysts using polymerase chain reaction on DNA isolated from faeces with spin columns. Eur J Clin Microbiol Infect Dis 19:358-361. doi:10.1007/s100960050494

27. Niesters HG (2002) Clinical virology in real time. J Clin Virol 25: S3-S12. doi:10.1016/S1386-6532(02)00197-X

28. Verweij JJ, Blangé RA, Templeton K, Schinkel J, Brienen EAT, van Rooyen MAA, van Lieshout L, Polderman AM (2004) Simultaneous detection of Entamoeba histolytica, Giardia lamblia, and Cryptosporidium parvum in fecal samples by using multiplex real-time PCR. J Clin Microbiol 42:1220-1223. doi:10.1128/ JCM.42.3.1220-1223.2004

29. Verweij JJ, Canales M, Polman K, Ziem J, Brienen EAT, Polderman AM, van Lieshout L (2009) Molecular diagnosis of Strongyloides stercoralis in faecal samples using real-time PCR. Trans R Soc Trop Med Hyg 103:342-346. doi:10.1016/j.trstmh.2008.12.001

30. Stark D, van Hal S, Fotedar R, Butcher A, Marriott D, Ellis J, Harkness J (2008) Comparison of stool antigen detection kits to PCR for diagnosis of amebiasis. J Clin Microbiol 46:1678-1681

31. Visser LG, Verweij JJ, van Esbroeck M, Edeling WM, Clerinx J, Polderman AM (2006) Diagnostic methods for differentiation of Entamoeba histolytica and Entamoeba dispar in carriers: performance and clinical implications in a non-endemic setting. Int $\mathrm{J}$ Med Microbiol 296:397-403. doi:10.1016/j.ijmm.2006.03.001
32. Siddiqui AA, Berk SL (2001) Diagnosis of Strongyloides stercoralis infection. Clin Infect Dis 33:1040-1047. doi:10.1086/ 322707

33. Bottieau E, Clerinx J, de Vega MR, Van den Enden E, Colebunders R, Van Esbroeck M, Vervoort T, Van Gompel A, Van den Ende J (2006) Imported Katayama fever: clinical and biological features at presentation and during treatment. J Infect 52:339-345. doi:10.1016/j.jinf.2005.07.022

34. ten Hove RJ, Verweij JJ, Vereecken K, Polman K, Dieye L, van Lieshout L (2008) Multiplex real-time PCR for the detection and quantification of Schistosoma mansoni and S. haematobium infection in stool samples collected in northern Senegal. Trans R Soc Trop Med Hyg 102:179-185. doi:10.1016/j.trstmh.2007. 10.011

35. Ten Hove RJ, van Lieshout L, Brienen EAT, Arantza Perez M, Verweij JJ (2008) Real-time polymerase chain reaction for detection of Isospora belli in stool samples. Diagn Microbiol Infect Dis 61:280-283. doi:10.1016/j.diagmicrobio.2008.03.003

36. Varma M, Hester JD, Schaefer FW 3rd, Ware MW, Lindquist HD (2003) Detection of Cyclospora cayetanensis using a quantitative real-time PCR assay. J Microbiol Methods 53:27-36. doi:10.1016/ S0167-7012(02)00209-9

37. Verweij JJ, Laeijendecker D, Brienen EA, van Lieshout L, Polderman AM (2003) Detection of Cyclospora cayetanensis in travellers returning from the tropics and subtropics using microscopy and real-time PCR. Int J Med Microbiol 293:199202. doi:10.1078/1438-4221-00252

38. Gushulak B, Funk M, Steffen R (2007) Global changes related to travelers' health. J Travel Med 14:205-208. doi:10.1111/j.17088305.2007.00128.x

39. Bailey MS, Thomas R, Green AD, Bailey JW, Beeching NJ (2006) Helminth infections in British troops following an operation in Sierra Leone. Trans R Soc Trop Med Hyg 100:842846. doi:10.1016/j.trstmh.2005.10.001

40. Schuurman T, de Boer RF, van Zanten E, van Slochteren KR, Scheper HR, Dijk-Alberts BG, Möller AV, Kooistra-Smid AM (2007) Feasibility of a molecular screening method for detection of Salmonella enterica and Campylobacter jejuni in a routine community-based clinical microbiology laboratory. J Clin Microbiol 45:3692-3700. doi:10.1128/JCM.00896-07

41. Mackay IM (2004) Real-time PCR in the microbiology laboratory. Clin Microbiol Infect 10:190-212. doi:10.1111/j.1198-743X. 2004.00722.x 